\title{
Odontólogos y Obligación de Denuncia del Abuso Sexual Infantil en Chile: Oportunidades Perdidas
}

\author{
Dentists and Mandatory Reporting of Child \\ Sexual Abuse in Chile: Missed Opportunities
}

\author{
Gabriel M. Fonseca'; Natalia Letelier-Acevedo²; Rubén Lozano-Gómez³
}

FONSECA, G. M.; LETELIER-ACEVEDO, N. \& LOZANO-GÓMEZ, R. Odontólogos y obligación de denuncia del abuso sexual infantil en Chile: Oportunidades perdidas. Int. J. Odontostomat., 12(4):431-436, 2018.

RESUMEN: La obligación de denunciar representa la estrategia clave para abordar al abuso sexual infantil, y se encuentra justificada en la suposición de que la detección temprana ayudará a prevenir lesiones graves (incluso la muerte del niño), aliviará a las víctimas de tener que buscar ayuda por su propia cuenta, y potenciará la coordinación entre las respuestas legales, médicas y sociales. Entre los profesionales sanitaristas con esta obligación se encuentran los odontólogos, quienes se han visto firmemente comprometidos a nivel mundial en la detección, denuncia y la prevención del abuso sexual a partir de la asumida y demostrada presencia de lesiones en boca. Sin embargo, y aunque la legislación vigente manifiesta esta obligación de denuncia, los odontólogos en Chile aparentan no estar preparados ni conceptual ni técnicamente para asumir esta responsabilidad, con lo que este tipo de prueba jurídica se encuentra subvalorada, abordada mínimamente por otras disciplinas no idóneas para tal efecto y virtualmente desconocida en los medios judiciales. En resumidas cuentas, una "oportunidad perdida" en un escenario donde las profundas miradas críticas y el severo juicio social obligan a afinar la mirada y ajustar la totalidad de las herramientas disponibles para lograr suficiencia de la prueba.

PALABRAS CLAVE: abuso sexual infantil, odontología, suficiencia probatoria.

\section{INTRODUCCIÓN}

En marzo de 2018, El Mostrador reportaba que en 2017, el ilícito más denunciado con 1.594 casos, fue el de abuso sexual de menores de 14 años (con contacto corporal), seguido de presunto abuso sexual de menor de 14 años (con circunstancias de violación) y violación de menor de 14 años. El reporte continúa: "Entre otros ilícitos, se registraron igualmente 80 denuncias por estupro, 41 por producción de pornografía infantil, 22 por facilitación de la prostitución de menores y 44 por ofensas al pudor" (El Mostrador, 2018). Ya iniciando 2018, "La Tercera" ofrecía un duro titular: "Más de 12 mil menores han sufrido abusos sexuales o violación en Chile" (Dodds, 2012). Citando estadísticas de la Corporación por los Derechos Sexuales y Reproductivos, el artículo refiere que "12.267 niños, niñas y adolescentes chilenos han sufrido de violación y abusos sexuales entre los años 2012 y 2016" (Dodds).

Los delitos sexuales son aquellos actos que atentan contra la libertad y la indemnidad sexual de las personas. El delito de violación está contemplado en el Código Penal de Chile haciéndose una distinción entre violación propia (artí- culo 361), la producida mediante el acceso carnal por vía vaginal, anal o bucal de víctimas mayores de 14 años en contra de su voluntad o aprovechando circunstancias de indefensión, e impropia (artículo 362), cuando en ese acceso se victimiza a menores de 14 años, aunque no concurra circunstancia alguna de las enumeradas antes. Respecto al abuso sexual, los artículos 366 (abuso propio) y 366 bis (abuso impropio) delimitan este delito a cualquier acción sexual distinta del acceso carnal, mientras el artículo 366 ter explicita que, para efectos de los artículos anteriores, "se entenderá por acción sexual cualquier acto de significación sexual y de relevancia realizado mediante contacto corporal con la víctima, o que haya afectado los genitales, el ano o la boca de la víctima, aún cuando no hubiere contacto corporal con ella". Cabe destacar que en Chile, el "abuso infantil", no existe como delito propiamente tal, por lo que para efectos de este trabajo, denominaremos "abuso sexual infantil" a los delitos sexuales realizados en contra de menores de 14 años, es decir, violación impropia o abuso sexual impropio y por ende el término será utilizado para denominar cualquier delito sexual realizado a estos menores de edad (Código Penal de Chile, 1874).

\footnotetext{
${ }^{1}$ Equipo para la Detección del Abuso y Maltrato Infantil (EDAMI), Centro de Investigación en Odontología Legal y Forense (CIO), Facultad de Odontología, Universidad de La Frontera. Temuco, Chile.

2 Defensoría Penal Pública. Santiago, Chile.

${ }^{3}$ Instituto San Juan de Estudios Superiores. Zacatecas, México.
} 
El Observatorio Nacional de los Derechos de la Niñez, expuso en 2017 una realidad preocupante: desde 2005 a 2012, el abuso sexual contra niñas, niños y adolescentes tuvo una tendencia al alza alcanzando un máximo de 131,3 niños y niñas víctimas por cada 100 mil habitantes en 2012. En los casos de violación, la evolución ha sido semejando a la del abuso sexual, alcanzando el mayor valor en 2011 (un promedio 28,1). El mismo informe revela que, según la única estadística provista en 2014 por el Servicio Médico Legal (SML), 5.024 niñas, niños y adolescentes fueron examinados por violación, abuso sexual u otro tipo de delito sexual. El informe concluye que "podría existir un porcentaje de casos encubiertos, lo que lleva a redoblar los esfuerzos en profundizar en esta materia" (Consejo Nacional de la Infancia, 2017).

En Chile, el Ministerio de Salud constituyó en 1991 la primera comisión para tratar el tema del abuso y maltrato infantil; cuatro años más tarde se creó el Comité Nacional de Prevención de Maltrato Infantil en el seno del Ministerio de Justicia, cuyos esfuerzos estuvieron centrados inicialmente en el ámbito legal, a posteriori ponderando las acciones preventivas, asistenciales y de capacitación (Moltedo \& Miranda, 2004). El deber de denunciar hechos con características de maltrato infantil o cualquier delito, se encuentra descrito tanto en la Ley de Menores como en el Código Procesal Penal y el Estatuto Administrativo, los que establecen obligación para los funcionarios/as públicos, directores/as de establecimientos educacionales públicos o privados y profesores/as, de denunciar estos hechos, en un plazo de 24 hs. a las que se tuvo conocimiento de los hechos, sancionándose su incumplimiento en el Artículo 177 del Código Procesal Penal Chileno.

La obligación de denunciar representa la oportunidad para abordar al abuso y maltrato infantil, y se encuentra justificada en que la detección temprana evitará lesiones graves (incluso la muerte del niño), aliviará a las víctimas de tener que buscar ayuda por su propia cuenta, y potenciará la coordinación entre las respuestas legales, médicas y sociales (Pietrantonio et al., 2013). En Chile, las estrategias de denuncia han sido concebidas sobre la reflexión, educación y prevención de las situaciones de abuso con especial atención tanto a las manifestaciones físicas como psicológicas del niño vulnerado. La entrada en vigencia de la Ley de Subvenciones $N^{\circ} 20.032$, ha permitido al Servicio Nacional de Menores (SENAME) el desarrollar programas de protección, todos originados en una primera y necesaria judicialización del evento de abuso y maltrato, postulando como "relevante" la coordinación con el Ministerio Público. Esto pretende acompañar de manera apropiada cada una de las etapas del proceso judicial: declaraciones de víctimas, peritajes, declaraciones de testigos, intervención en crisis con la víctima y su familia durante el juicio, etc. (Servicio Nacional de Menores, 2012). Por su parte, el Ministerio Público de Chile ha formalizado su "Protocolo de Actuación ante la sospecha o denuncia de abuso sexual contra un menor de edad" (Fiscalía del Ministerio Público de Chile, 2013), documento cuyo objeti- vo es dar forma a una denuncia apropiada para "potenciar el conocimiento de la Fiscalía de este tipo de situaciones y facilitar la acreditación de los hechos ante los tribunales". Para los primeros meses de 2015, y según cifras reportadas por el SENAME, de las más de 25 mil denuncias realizadas por delitos sexuales, unas 18 mil correspondieron a delitos sexuales contra niños, niñas y adolescentes. Sólo 2 mil casos terminaron en sentencia condenatoria; el tiempo de tramitación (en días) de juicios orales por delitos sexuales tuvo un promedio nacional de más de dos años, con un máximo en Región Metropolitana Centro Norte (930 días) y un mínimo en la IX Región (490 días) (Servicio Nacional de Menores, 2015).

Todas las definiciones que existen de abuso sexual infantil tienen en común tres factores: a) un niño/a involucrado en actividades sexuales; b) agresor en una posición de poder y control sobre el niño/a; c) maniobras de coerción por parte del abusador: seducción, manipulación y amenaza. Kempe define el abuso sexual como "implicación de un niño (a) o de un adolescente menor en actividades sexuales ejercidas por un adulto y que buscan la satisfacción de él, siendo los menores de edad inmaduros y dependientes y por tanto incapaces de comprender el sentido radical de estas actividades, ni por tanto de dar su consentimiento real. Estas actividades son inapropiadas a su edad y a su nivel de desarrollo psicosexual y son impuestas bajo presión-por la violencia o seducción- y trasgreden tabúes sociales en lo que concierne a los roles familiares" (Venegas González, 2003). La legislación chilena sobre delitos sexuales, los clasifica en violación, incesto, estupro, sodomía, abuso sexual calificado, pornografía infantil y utilización de niños para la prostitución. Las sanciones de mayor gravedad se encuentran previstas en casos de víctimas menores de edad, ampliando la sanción cuando los perpetradores son personas vinculadas a las víctimas, incluso establecida una relación directa o habitual con menores de edad. El acceso carnal, concepto entendido para la doctrina nacional mayoritaria como la introducción del pene-excluidos objetos u otras prolongaciones corporales- logra a través de su mención en la Ley 19.617 establecer con precisión cuáles son las vías de acceso en la violación: anal, vaginal y bucal (Biblioteca del Congreso Nacional de Chile, 1999). Sobre esta última, y sólo considerando el criterio del inmissio penis aceptado en la doctrina chilena (Rodríguez Collao, 2014) para designar "la introducción del pene en la boca" (evitando con ello el profundizar en otras conductas atípicas), este estudio pretende responder tres preguntas esenciales: 1) ¿Existen lesiones en la boca que puedan relacionarse con abuso sexual infantil específicamente?, 2) ¿Qué profesional de la salud es el más competente para evaluarlas? y 3) ¿Cuál es la situación real/ideal respecto del deber de denuncia de estos delitos en estos profesionales?

Lesiones Orales, Odontología, y la Denuncia de Abuso Sexual Infantil. No existen dudas de que un delito sexual implica una forma de contacto entre un perpetrador y una víctima de cualquier sexo o edad, y que entre ellos deberá 
suscitarse una interacción corporal o mediante instrumentos con significación sexual a través del uso de violencia física y/o psicológica. También hay certezas de que la figura de abuso podrá aplicarse cuando este acto es ejercido a través del sometimiento o haciendo uso de una posición de poder o control sobre la víctima. Sin embargo, objetivar este contacto no siempre es posible (Fondo de las Naciones Unidas para la Infancia, 2006); más allá de los evidentes cambios en el comportamiento de la víctima, la práctica recomendada de reconocer las señales físicas resultantes de ese abuso, puede ser muy dificultosa (Fiscalía del Ministerio Público de Chile).

A nivel mundial, la profesión odontológica se ha visto firmemente comprometida en la detección, denuncia y prevención del abuso infantil (Cukovic-Bagic et al., 2013), asumiendo una responsabilidad sobre bases que han demostrado que entre el 50 y el $75 \%$ de los casos de niños abusados poseen heridas en su boca, cara y cabeza (Bsoul et al., 2003). La última revisión 2017 de la "Guía sobre aspectos oral y dental del abuso y maltrato infantil" propuesta por el Comité de Abuso y Maltrato Infantil con miembros de las Academias Americanas de Pediatría y de Odontopediatría, concluyó que es importante que los prestadores de salud (incluyendo odontólogos), consideren que el abuso físico o sexual puede provocar lesiones en tejidos orales. Estos deben conocer cuándo y cómo documentar lesiones sospechosas y cómo obtener evidencia de laboratorio, documentación fotográfica y/o consultar con expertos cuando corresponda (Fisher-Owens et al., 2017). Ese documento revisa minuciosamente cuadros clínicos como mordeduras, heridas orales, infecciones y enfermedades que pueden ser manifestación de negligencia, abuso físico o abuso sexual infantil. Sobre este último particular, el documento Ilama la atención sobre lesiones "patognomónicas" de abuso sexual -particularmente lesiones por gonorrea oral o perioral-, y sobre otras fuertemente inductoras de delitos sexuales, las que deben ser convenientemente examinadas y diagnosticadas para su confirmación: la presencia de algunas formas específicas de verrugas, o el hallazgo de particulares enrojecimientos en el paladar, todas consecuentes -pero no exclusivas- de contacto orogenital (Fisher-Owens et al.). La descriptiva de este universo de imágenes clínicas no es nueva ni azarosa (Fonseca, 2012, 2014) y la odontología mundial se ha mantenido atenta no sólo a su detección sino también a la concientización de los odontólogos para asumir un rol protagónico (Fonseca, 2012; Rai \& Kaur, 2013).

Sin embargo, las diferentes asociaciones odontológicas han recomendado la utilización de estándares estrictos y prudentes para estos fines (Bsoul et al.). En el caso específico de las denominadas "Enfermedades de Transmisión Sexual" (ETS), si bien el diagnóstico mediante exámenes de laboratorio puede ser sencillo, y acuciosa la denuncia según las recomendaciones vigentes (Menni, 2007)-, establecer con seguridad su origen en un contacto bucogenital implica recorrer un camino mucho más complejo; en niños, las ETS orales por transmisión no sexual o accidental, han sido ampliamente reportadas (Villanueva, 2010). La literatura médica recomienda en estos casos mesura y suficiencia antes de efectuar la denuncia pues una equivocación a estos niveles puede tener efectos devastadores tanto en la supuesta "víctima" como en su familia (Narang et al., 2013). Este "umbral de suficiencia" parece tener efectos frustrantes. Países de todo el globo han informado que sus odontólogos no reconocen signos de abuso ni saben cómo hacer las denuncias respectivas (Bsoul et al.; Al-Habsi et al., 2009; Owais et al., 2009; Uldum et al., 2010; Harris et al., 2013; Laud et al., 2013). En Latinoamérica la información es todavía más escasa pero igualmente decepcionante (Arrieta Vergara et al., 2014); los odontólogos brasileños (incluso odontopediatras), no se sienten capacitados para identificar abuso ni saben dónde hacer las denuncias. Casi la totalidad afirma que el conocimiento de abuso infantil recibido en su educación de grado fue notoriamente insuficiente (Andrade et al., 2005).

La odontología chilena parece seguir el mismo camino. Mientras que el Código Procesal Penal, y específicamente el inciso "d" del artículo 175 incluye a los odontólogos entre los profesionales obligados a denunciar en caso de detectar signos de este tipo de delitos (Código Procesal Penal de Chile, 2000), un estudio publicado por académicos de la Facultad de Odontología de la Universidad de Concepción en 2012 (Fierro Monti et al., 2012) concluyó que los odontólogos no están preparados para identificar abuso infantil. Más de la mitad de los 58 profesionales encuestados reconoció haber tenido sospechas de que alguno de sus pacientes había sido abusado o maltratado, pero sólo 13 hicieron las denuncias correspondientes. Ortodoncistas y odontopediatras (quienes atienden casi de manera exclusiva a niños) revelaron conocimientos inferiores al promedio, con lo que los autores destacan la necesaria incorporación del tema en el pregrado de las facultades de odontología. El $66 \%$ de los odontólogos encuestados no reconoció que las petequias en el paladar pueden ser signo clínico de sexo oral forzado (aseveración número 10) (Fierro Monti et al.). Nuestras observaciones hacen eco de estas circunstancias; en actividades de capacitación brindadas a funcionarios de la Brigada Investigadora de Delitos Sexuales y Menores Temuco (BRISEXME) de la Policía de Investigaciones (PDI) (Fig. 1), los funcionarios afirmaron desconocer denuncias formales iniciadas por odontólogos en este ámbito. En 2015, una Comisión de la Asociación Chilena de Enseñanza en Odontología, luego de analizar las programas provistos por profesores que imparten la asignatura de pregrado "Odontología Legal" en once universidades chilenas, informó que el tópico "Abuso y Maltrato Infantil", competencia innegable de ese ramo según los estándares internacionales (Hinchliffe, 2013), es abordado sólo por una facultad de odontología en todo Chile (Asociación Chilena de Enseñanza de la Odontología, 2015).

Un dato curioso: el mencionado "Protocolo de actuación ante la sospecha o denuncia de abuso sexual contra un menor de edad", documento propuesto por el Ministerio 
Público para "potenciar el conocimiento de la Fiscalía de este tipo de situaciones y facilitar la acreditación de los hechos ante los tribunales", menciona la presencia de semen en la boca como único signo físico oral perceptible y "altamente sugerente" de abuso sexual por esa vía (Fiscalía del Ministerio Público de Chile). Sin lugar a dudas una "simplista" manera de dar claridad y suficiencia al hecho. Resulta importante contextualizar esa información; el mismo protocolo previamente ha dejado entrever que "las señales físicas del abuso suelen aparecer en una parte muy pequeña de casos confirmados...", algo ya informado por la literatura científica quien prima la posibilidad de encontrar mayores porcentajes de perturbaciones psicológicas sobre las físicas (Almonte et al., 2002). Sin embargo, resulta interesante la percepción del Comité de Abuso y Maltrato Infantil de la Academia Americana de Pediatría: aunque destacan esas enormes dificultades diagnósticas, los autores prefieren hablar de "oportunidades perdidas" cuando los profesionales no han hecho un manejo apropiado de lesiones menores pero sospechosas -ellos las llaman lesiones "centinela"-, presentes en un $25 \%$ de los niños abusados (Christian \& Committee on Child Abuse and Neglect, American Academy of Pediatrics, 2015). Nuevamente desde la perspectiva del odontólogo, ya en 1986, Casamassimo afirmaba que, en interés del niño como motivador primario, la mejor manera de resolver este problema es asumir honestamente las falencias: los dentistas pueden no estar capacitados para reconocer las manifestaciones orales de enfermedades de transmisión sexual u otras lesiones sexuales orales (Casamassimo, 1986). Katner \& Brown (2012) afirman que para los odontólogos, el deber de denuncia ante sospecha de abuso infantil se fundamenta en tres razones: 1) existe obligación legal implícita cuyo incumplimiento puede tener sanciones criminales; 2) existe deber ético profesional para denunciar tales casos; 3 ) los odontólogos pueden ser además demandados civilmente por mala praxis por inobservancia de sus deberes, instancias sobre las que existe un claro acuerdo mundial.

\section{DISCUSIÓN Y CONCLUSIONES}

Objetivar el daño físico en el abuso sexual se logra en sólo un $5 \%$ de los casos (Ministerio de Salud de Chile, 2011); sin embargo este porcentaje toma relevancia si se consideran las estadísticas ya citadas (EI Mostrador; Dodds). El profesional de la salud debe estar capacitado "para ver lo invisible", utilizar guías de indicadores, y realizar exámenes físicos detallados en busca de lesiones (Ministerio de Salud de Chile). Entre las propuestas para disminuir la victimización secundaria, una de las más importantes la conforma la "Especialización de los profesionales involucrados en materia de infancia" (Servicio Nacional de Menores, 2015), esto referido a toda la gama de peritajes (incluidos los de testigos expertos) pues constituyen parte fundamental de la prueba que se presenta en los juicios por estos delitos; una mayor especia-

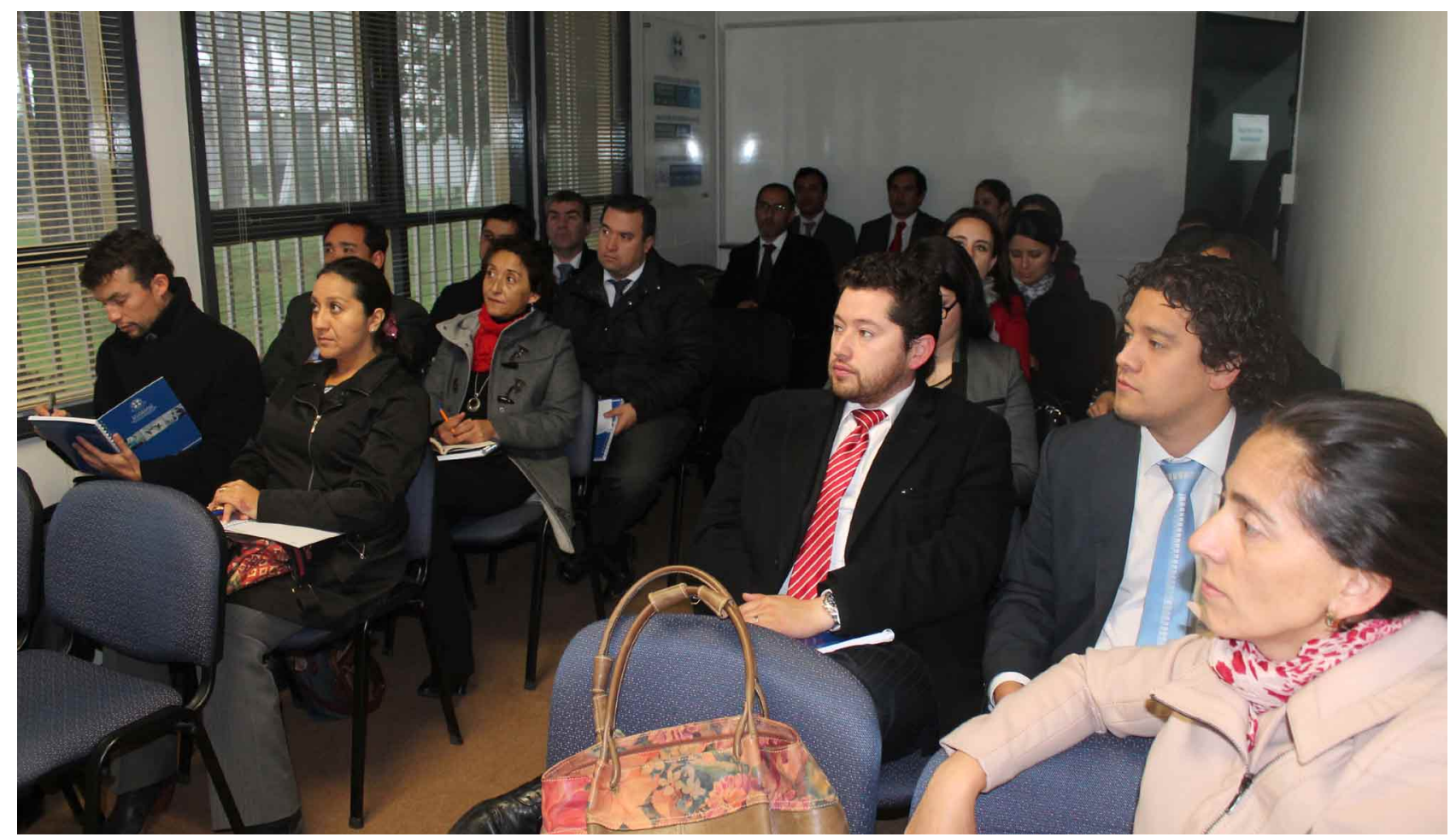

Fig. 1. Capacitación sobre lesiones orales por abuso y maltrato infantil impartida por el Laboratorio de Pericias en Odontología Forense (LPO) de la Facultad de Odontología de la Universidad de La Frontera a funcionarios de la BRISEXME Temuco, Policía de Investigaciones de Chile. Disponible en: www.odontologiaufro.cl/index.php/archivo-de-noticias/446-201505-12-20-12-05). 
lización permitiría no sólo la realización de peritajes oportunos y con una metodología definida, sino también el poder instalar nuevas estrategias judiciales que afiancen los medios probatorios para lograr una mayor cantidad de sentencias condenatorias. Según el SENAME, estas estrategias hoy son utilizadas raramente dado el bajo nivel de especialización de los profesionales que deben tratar casos de abuso sexual (Servicio Nacional de Menores, 2015).

Se ha subrayado que en odontología no sólo existen necesidades de capacitación en detección de abuso infantil tanto en el pre como en el postgrado, sino también que autoridades y organismos gubernamentales deberían hacer mayores esfuerzos para la construcción de un sistema más organizado (Laud et al.). Para lograr estos deberes profesionales, éticos y legales, los odontólogos deberían ser educados en tópicos relativos al abuso y maltrato infantil tanto en pre y postgrado (Cukovic-Bagic et al.). Esta preocupación no es aislada ni azarosa; a diferentes niveles pero con igual grado de compromiso, odontólogos en todo el mundo han reconocido estas vulnerabilidades y han generado coaliciones destinadas a brindar herramientas suficientes diagnósticas y de asesoría legal para la prevención del abuso infantil: PANDA (Prevent Abuse and Neglect Through Dental Awareness) en los Estados Unidos (con filiales en 44 estados), o BEAR (Basic Education for Abuse Recognition) en Canadá desarrollan intensas campañas de capacitación, con financiamiento institucional y gubernamental (Lincoln \& Lincoln, 2010). Trasladar estos estándares a nuestras latitudes implica fuertes compromisos de universidades, asociaciones científicas y entidades de acreditación (Ivanoff \& Hottel, 2013), responsabilidades hoy no asumidas a ningún nivel odontológico en Chile. Sin embargo, consideramos que las cargas no son de exclusiva competencia académica; visualizando esto como una tríada odontólogo/médico forense/juez, la insuficiencia de un odontólogo como efector de diagnóstico y denuncia ante lesiones orales por delitos sexuales, obliga al médico clínico y/o forense a tener que detectar señales sobre las que no ha sido capacitado (con el consiguiente subdiagnóstico) y al juez a dirimir fallos con la evidente falta de suficiencia de pruebas. El odontólogo como "testigo experto" en casos de abuso sexual infantil, representa hoy en Chile un recurso inexplorado, subvalorado y carente de promoción, aún siendo considerado como una de las piezas orgánicamente obligadas a asumir un rol de activo denunciante según la legislación vigente. En resumidas cuentas, una "oportunidad perdida" (Christian \& Committee on Child Abuse and Neglect, American Academy of Pediatrics) en un escenario donde las profundas miradas críticas y el severo juicio social obligan a afinar la mirada y ajustar la totalidad de las herramientas probatorias disponibles.

FONSECA, G. M.; LETELIER-ACEVEDO, N. \& LOZANO-GÓMEZ, R. Dentists and mandatory reporting of child sexual abuse in Chile: missed opportunities. Int. J. Odontostomat., 12(4):431-436, 2018.

ABSTRACT: Mandatory reporting represents the key strategy in child sexual abuse cases. It is based on the assumption that early detection will help prevent serious injuries (even the death of the child), will alleviate victims from the responsibility of asking for help themselves, and at their own risk, and will also encourage coordination between legal, medical, and social response units. Among health professionals with a reporting obligation are odontologists. Worldwide, dentists have actively committed to take part in the detection, reporting and prevention of child sexual abuse from presumed and proven lesions in the mouth. However, although current legislation explicitly states this mandatory reporting, it appears that Chilean dentists are neither conceptually nor technically prepared to take on this responsibility. Hence, this type of forensic legal evidence is undervalued, inadequately addressed within other medical professions, and practically unknown in the judicial system. In summary, this is a "missed opportunity" in circumstances that warrant serious scrutiny, resulting in severe social judgment and criticism. A complete review and regulation of the guidelines in this area must be carried out in order to use all means available, and apply legal measures to provide the necessary proof in these cases.

proof.

KEY WORDS: child sexual abuse, odontology, sufficient

\section{REFERENCIAS BIBLIOGRÁFICAS}

Al-Habsi, S. A.; Roberts, G. J.; Attari, N. \& Parekh, S. A survey of attitudes, knowledge and practice of dentists in London towards child protection. Are children receiving dental treatment at the Eastman Dental Hospital likely to be on the child protection register? Br. Dent. J., 206(4):E7; discussion 212-3, 2009.

Almonte, C.; Insunza, C. \& Ruiz, C. Abuso sexual en niños y adolescentes de ambos sexos. Rev. Chil. Neuro-Psiquiatr., 40(1):2230,2002

Andrade, L. K.; Colares, V. \& Cabral, H. M. Avaliação da conduta dos odontopediatras de Recife com relação ao abuso infantil. Rev. Odonto. Ciênc., 20(49):231-6, 2005.

Arrieta Vergara, K. M.; Díaz Cárdenas, S. \& González Martínez, F. Maltrato infantil: conocimientos, actitudes y prácticas de estudiantes de Odontología de Cartagena, Colombia. Rev. Clin. Med. Fam., 7(2):103-11, 2014.

Asociación Chilena de Enseñanza de la Odontología. Comisión Revisión de Contenidos de Pregrado Área Odontología Legal. Recomendaciones de Contenidos Mínimos Curriculares Área Odontología Legal. Informe Final. Santiago de Chile, Asociación Chilena de Enseñanza de la Odontología, 2015.

Biblioteca del Congreso Nacional de Chile. Historia de la Ley $N^{\circ}$ 19.617. Modifica el Código Penal, el Código de Procedimiento Penal y otros cuerpos legales, en materias relativas al delito de violación. Santiago de Chile, Biblioteca del Congreso Nacional de Chile, 1999. Disponible en: www.leychile.cl/navegar/scripts/ obtienearchivo?id=recursoslegales/10221.3/3848/1/hl19617.pdf

Bsoul, S. A.; Flint, D. J.; Dove, S. B.; Senn, D. R. \& Alder, M. E. Reporting of child abuse: a follow-up survey of Texas dentists. Pediatr. Dent., 25(6):541-5, 2003.

Casamassimo, P. S. Child sexual abuse and the pediatric dentist. Pediatr. Dent., 8(1 Spec. No.):102-6, 1986.

Christian, C. W. \& Committee on Child Abuse and Neglect, American Academy of Pediatrics. The evaluation of suspected child physical abuse. Pediatrics, 135(5):e1337-54, 2015.

Código Penal de Chile. 12 de noviembre de 1874 y modificaciones subsiguientes. Santiago de Chile, Ministerio de Justicia, 1874

Código Procesal Penal de Chile. Ley No 19.696, 12 de octubre de 2000. Artículos 175 a 179. Santiago de Chile, Ministerio de Justicia, 2000. 
Consejo Nacional de la Infancia. Situación de los Derechos de Niños, Niñas y Adolescentes en Chile 2017, Segundo Informe. Santiago de Chile, Consejo Nacional de la Infancia, 2017. pp.137-9. Disponible en: http://observatorioninez.consejoinfancia.gob.cl/wp-content/ uploads/2018/02/Informe-Monitoreo-Derechos-2017-2.pdf

Cukovic-Bagic, I.; Welbury, R. R.; Flander, G. B.; Hatibovic-Kofman, S. \& Nuzzolese, E. Child protection: legal and ethical obligation regarding the report of child abuse in four different countries. $J$. Forensic Odontostomatol., 31(1):15-21, 2013.

Dodds, T. Más de 12 Mil Menores han Sufrido Abusos Sexuales o Violación en Chile desde el 2012. La Tercera, edición del 2 de enero de 2018. Santiago de Chile, La Tercera, 2018. Disponible en: http:// www2.latercera.com/noticia/mas-12-mil-menores-sufrido-abusossexuales-violacion-chile-desde-2012/

El Mostrador. Más de 4 mil 200 denuncias por abuso sexual recibió la PDI durante 2017. Edición del 6 de marzo de 2018. Santiago de Chile, El Mostrador, 2018. Disponible en: www.elmostrador.cl/noticias/pais/2018/03/06/casi-5-mil-denuncias-por-abuso-sexual-recibiola-pdi-durante-2017|

Fierro Monti, C.; Salazar Salazar, E.; Ruiz Salazar, L.; Luengo Machuca, L. \& Pérez Flores, A. Attitude towards child abuse according to the knowledge of dentists in Concepción, Chile. Int. J. Odontostomat., 6(1):105-10, 2012.

Fiscalía del Ministerio Público de Chile. Protocolo de Actuación ante la sospecha o denuncia de abuso sexual contra un menor de edad. Santiago de Chile, Fiscalía del Ministerio Público de Chile, 2013. Disponible en: www.fiscaliadechile.cl/Fiscalia/ archivo?id=4241\&pid=1601\&tid=2\&d=1

Fisher-Owens, S. A.; Lukefahr, J. L.; Tate, A. R.; American Academy of Pediatrics, Section on Oral Health; Committee on Child Abuse and Neglect; American Academy of Pediatric Dentistry, Council on Clinical Affairs, Council on Scientific Affairs \& Ad Hoc Work Group on Child Abuse and Neglect. Oral and dental aspects of child abuse and neglect. Pediatrics, 140(2):e20171487, 2017.

Fondo de las Naciones Unidas para la Infancia (Oficina para Chile y el Conosur), Instituto de Investigación en Ciencias Sociales - Universidad Diego Portales. Niños, Niñas y Adolescentes Víctimas de Delitos Sexuales, en el Marco de la Reforma Procesal Penal. Informe Final. Santiago de Chile, Fondo de las Naciones Unidas para la Infancia, 2006. Disponible en: www.unicef.cl/web/wp-content/ uploads/doc_wp/Informe\%20final.pdf

Fonseca, G. M. Eritema en paladar por felación. Revisión a propósito de un caso. Rev. Tamé, 1(1):19-23, 2012.

Fonseca, G. M. Felación Forzada. En: Meriño Aravena, M. (Ed.). Los Delitos Sexuales desde una Perspectiva Interdisciplinaria. Santiago de Chile, Ediciones Jurídicas de Santiago, 2014. pp.161-95.

Harris, C. M.; Welbury, R. \& Cairns, A. M. The Scottish dental practitioner's role in managing child abuse and neglect. Br. Dent. J., 214(9):E24, 2013.

Hinchliffe, J. Forensic odontology, part 5. Child abuse issues. Br. Dent. J., 210(9):423-8, 2013.

Ivanoff, C. S. \& Hottel, T. L. Comprehensive training in suspected child abuse and neglect for dental students: a hybrid curriculum. J. Dent. Educ., 77(6):695-705, 2013.

Katner, D. R. \& Brown, C. E. Mandatory reporting of oral injuries indicating possible child abuse. J. Am. Dent. Assoc., 143(10):1087-92, 2012.

Laud, A.; Gizani, S.; Maragkou, S.; Welbury, R. \& Papagiannoulis, L. Child protection training, experience, and personal views of dentists in the prefecture of Attica, Greece. Int. J. Paediatr. Dent., 23(1):6471, 2013.

Lincoln, H. S. \& Lincoln, M. J. Role of the odontologist in the investigation of domestic violence, neglect of the vulnerable, and institutional violence and torture. Forensic Sci. Int., 201(1-3):68-73, 2010.

Menni, S. Acquired primary syphilis on a child's lip. Acta Derm. Venereol., 87(3):284, 2007.

Ministerio de Salud de Chile; Fondo de las Naciones Unidas para la
Infancia \& UNICEF. Guía clínica para la atención de niños, niñas y adolescentes menores de 15 años víctimas de abuso sexual. Santiago de Chile, Ministerio de Salud de Chile, 2011.

Moltedo, C. \& Miranda, M. Protegiendo los Derechos de Nuestros Niños y Niñas. Prevención del Maltrato y el Abuso Sexual Infantil en el Espacio Escolar. Manual de Apoyo para Profesores. Santiago de Chile, Ministerio de Justicia, Fundación de la Familia, 2004. p.94.

Narang, T.; Kanwar, A. J. \& Kumaran, M. S. Condyloma lata in a preschooler: The dilemma of sexual abuse versus non-abuse. Indian J. Sex Transm. Dis., 34(2):135-7, 2013.

Owais, A. I.; Qudeimat, M. A. \& Qodceih, S. Dentists' involvement in identification and reporting of child physical abuse: Jordan as a case study. Int. J. Paediatr. Dent., 19(4):291-6, 2009.

Pietrantonio, A. M.; Wright, E.; Gibson, K. N.; Alldred, T.; Jacobson, D. \& Niec, A. Mandatory reporting of child abuse and neglect: crafting a positive process for health professionals and caregivers. Child Abuse Negl., 37(2-3):102-9, 2013.

Rai, B. \& Kaur, J. Role of Dentist and Forensic Odontologist in Child Abuse Cases. En: Rai, B. \& Kaur, J. (Eds.). Evidence-Based Forensic Dentistry. Berlín,Springer-Verlag, 2013. p.180.

Rodríguez Collao, L. Delitos Sexuales. $2^{\mathrm{a}}$ ed. Santiago de Chile, Editorial Jurídica de Chile, 2014.

Servicio Nacional de Menores (SENAME). Bases Técnicas, Línea Programas de Protección Especializada en Maltrato y Abuso Sexual Infantil (PRM). Santiago de Chile, Servicio Nacional de Menores, 2012. Disponible en: www.sename.cl/wsename/licitaciones/p32-1811-2013/7_BASES_TECNICAS_PRM.pdf

Servicio Nacional de Menores (SENAME). Justicia proporcional a los niños, niñas y adolescentes. Discusión Proyecto de Ley que Regula entrevistas grabadas en video y otras medidas de resguardo a menores de edad víctimas de abuso sexual. Santiago de Chile, Servicio Nacional de Menores, 2015. Disponible en: www.senado.cl/ appsenado/index.php? $\mathrm{mo}=$ tramitacion $\& a c=$ getDocto\&iddocto =963\&tipodoc=docto_comision

Uldum, B.; Christensen, H. N.; Welbury, R. \& Poulsen, S. Danish dentists' and dental hygienists' knowledge of and experience with suspicion of child abuse or neglect. Int. J. Paediatr. Dent., 20(5):3615, 2010.

Venegas González, S. Labor Pericial en Delitos Sexuales contra Menores. Unidad de Maltrato y Psiquiatría Infantil, Departamento de Clínica Forense. Santiago de Chile, Servicio Médico Legal, 2003.

Villanueva, J. C. Lesiones asociadas con HPV en mucosa oral de niños: ¿prueba inequívoca de abuso? F. O. P. J., 1(1):12-5, 2010.

Dirección para correspondencia:

Dr. Gabriel M. Fonseca

Equipo para la Detección del Abuso y Maltrato Infantil (EDAMI), Centro de Investigación en Odontología Legal y Forense, Edificio L

Facultad de Odontología

Universidad de La Frontera

Francisco Salazar 01145

Temuco

CHILE

Email: gabriel.fonseca@ufrontera.cl

Recibido : 17-07-2018

Aceptado: 07-09-2018 